\title{
Revascularização do miocárdio sem circulação extracorpórea: resultados da experiência de 18 anos de sua utilização
}

\author{
Luciano F. AGUIAR*, José Carlos S. ANDRADE*, João Nelson BRANCO*, José Honório PALMA*, \\ Carlos A. TELES*, Luis Roberto GEROLA*, Ênio BUFFOLO*
}

RBCCV 44205-523

\begin{abstract}
Aguiar L F, Andrade J C S, Branco J N, Palma J H, Teles C A, Gerola L R, Buffolo E - Revascularização do miocárdio sem circulação extracorpórea: resultados da experiência de 18 anos de sua utilização. Rev Bras Cir Cardiovasc 2001; 16(1): 1-6.
\end{abstract}

RESUMO: Introdução: A revascularização do miocárdio sem circulação extracorpórea (CEC) é hoje alternativa de revascularização em expansão não estando ainda bem definidos os limites de sua aplicabilidade. Os autores revêem sua casuística global e discutem as indicações ideais do procedimento baseados nos resultados obtidos.

Casuística e Métodos: São analisados 2.495 pacientes operados sem CEC de outubro de 1981 a setembro de 1999 de um total de 10.656 pacientes revascularizados no período $(23,4 \%)$. As idades variaram de 32 a 90 anos, mediana 59, e o sexo masculino predominou (67\%). Insuficiência coronária crônica foi responsável por $70,8 \%$ das indicações operatórias e a maioria dos pacientes recebeu 2 pontes $(51,5 \%)$.

Resultados: A mortalidade (30 dias) global foi de 1,9\% (48/2495) e a morbidade referente a eventos maiores foi significativamente menor em um subgrupo deste material que foi prospectivamente. Apenas $45 \%$ dos operados necessitaram de transfusão sangüínea contrastando com $90,5 \%$ dos operados com a técnica convencional. A aplicabilidade da técnica que no global foi de $23 \%$ nos últimos três anos foi de $36,8 \%, 35$, $1 \%$ e $42,2 \%$.

Conclusão: A revascularização do miocárdio sem CEC é alternativa segura de tratamento da insuficiência coronária observando-se um aumento progressivo de sua aplicação nos últimos anos. Permite o tratamento de um subgrupo de pacientes com baixa mortalidade hospitalar e menor incidência de complicações pós-operatórias, devendo seu uso ser expandido nos próximos anos com a introdução dos estabilizadores, manobras especiais e a revascularização funcional.

DESCRITORES: Revascularização miocárdica, métodos. Circulação extracorpórea. Baixo débito cardíaco, cirurgia. Procedimentos cirúrgicos minimamente invasivos. Procedimentos cirúrgicos cardíacos, métodos.

Trabalho realizado na Disciplina de Cirurgia Cardiovascular da Escola Paulista de Medicina da Universidade Federal de São Paulo. São Paulo, SP, Brasil.

Apresentado ao 27ํㅡ Congresso Nacional de Cirurgia Cardíaca. Rio de Janeiro, RJ, 23 a 25 de março de 2000.

* Da Escola Paulista de Medicina

Endereço para correspondência: Luciano F. Aguiar. Rua Borges Lagoa, 783, 5ํandar, São Paulo, SP, Brasil. CEP 04038-031. Tel: (011) 573-3009.

Fax: (011) 573-0303. e-mail: cardiovascular.dcir@epm.br 
Aguiar L F, Andrade J C S, Branco J N, Palma J H, Teles C A, Gerola L R, Buffolo E - Revascularização do miocárdio sem circulação extracorpórea: resultados da experiência de 18 anos de sua utilização. Rev Bras Cir Cardiovasc 2001; 16(1): 1-6.

\section{INTRODUÇÃO}

A revascularização direta do miocárdio sem o uso de circulação extracorpórea foi de forma inédita realizada por KOLESSOV (1), na União Soviética, seguido por FAVALORO (2) e GARRET et al.(3), nos Estados Unidos.

No Canadá, TRAPP \& BISARYA (4), ao mesmo tempo ANKENEY ${ }^{(5)}$, nos Estados Unidos, também foi realizada a técnica precocemente que foi abandonada devido à extracorpórea apresentar grande facilidade e conforto no momento da realização de anastomoses com o coração sem movimentos durante a parada anóxica.

A partir de outubro de 1981, passamos a realizar de forma sistemática anastomose mamária e safena, coronária preferencialmente, para as artérias descendente anterior, diagonal e coronária direita com interrupção temporária do fluxo coronário, manobras locais e uso de drogas que diminuem o consumo de $\mathrm{O}_{2}$ pelo miocárdio.

Os resultados iniciais foram animadores e nos entusiasmaram a aprimorar nossos resultados (6.7), bem como outros autores como RIVETTI et al. (8), em 1997, utilizando shunt intracardíaco temporário sem interromper fluxo coronário e JATENE et al. ${ }^{(9)}$ e LOBO FILHO et al. (10), ampliando em muito a utilização do método.

Também divulgamos os principais critérios de indicação do procedimento, assim como sua comparação com procedimento convencional $(11,12)$.

A introdução da utilização de minitoracotomia esquerda, vídeo assistência, auxílio de estabilizadores $(13,14)$ tornam, hoje, uma alternativa de revascularização em expansão e que ainda necessita definir os limites de sua aplicabilidade.

Baseados em nossos resultados, nos pronomes a discutir as indicações ideais para o procedimento.

\section{CASUÍSTICA E MÉTODOS}

De outubro de 1981 a setembro de 1999, foram submetidos a revascularização do miocárdio um total de 10656 pacientes sendo 2495 (23\%) pacientes sem o uso da circulação extracorpórea (Tabela 1).

A via de acesso utilizada foi esternotomia mediana limitada em $93,3 \%$ dos pacientes e em $6,7 \%$ foi utilizada a toracotomia mínima anterior esquerda para paciente com lesões isoladas em descendente anterior ou em associação com a diagonal (Tabela 2).

O número de pontes variou de 1 a 5 , sendo que a maioria dos pacientes $(51,5 \%)$ recebeu 2 pontes
TABELA 1

\begin{tabular}{lc}
\hline \multicolumn{2}{c}{ REVASCULARIZAÇÃO DO MIOCÁRDIO SEM CEC } \\
APLICABILIDADE \\
\hline GLOBAL & $\mathbf{2 4 9 5} / \mathbf{1 0 6 5 6}(\mathbf{2 3 \% )}$ \\
1997 & $36,8 \%$ \\
1998 & $35,1 \%$ \\
1999 & $42,2 \%$ \\
\hline
\end{tabular}

TABELA 2

REVASCULARIZAÇÃO DO MIOCÁRDIO SEM CEC

CASUÍSTICA

\begin{tabular}{ll}
\hline Esternotomia Mediana Limitada & $93,3 \%$ \\
Minitoracotomia Esquerda & $6,7 \%$ \\
\hline
\end{tabular}

TABELA 3

REVASCULARIZAÇÃO DO MIOCÁRDIO SEM CEC TIPOS DE ENXERTO

\begin{tabular}{ll}
\hline Enxertos Arteriais e Safena & $65 \%$ \\
Somente Enxertos Arteriais & $20 \%$ \\
Somente Safena & $15 \%$ \\
TOTAL & $100 \%$ \\
\hline
\end{tabular}

por procedimento, com média de 1,9 pontes por paciente (Tabela 3).

Com relação ao tipo de enxerto utilizado, empregamos, na maioria dos casos, artérias associadas com veia safena em $65 \%$, somente arteriais (mamária, radial ou gastro-omental) em 20\% e somente venoso em $15 \%$ deste total (Gráfico 1).

As condições clínicas que indicaram a cirurgia foram, na sua maior incidência, a insuficiência coronária crônica e eletiva $(69,3 \%)$. Porém, em um grande porcentual $(20,7 \%)$, foram submetidos a cirurgia em condições especiais ou por vezes em urgências, inclusive com utilização de balão intraaórtico como suporte em 18 pacientes (Tabela 4).

A técnica operatória utilizada na revascularização do miocárdio sem circulação extracorpórea já foram descritas com detalhes em publicações anteriores. 
Aguiar L F, Andrade J C S, Branco J N, Palma J H, Teles C A, Gerola L R, Buffolo E - Revascularização do miocárdio sem circulação extracorpórea: resultados da experiência de 18 anos de sua utilização. Rev Bras Cir Cardiovasc 2001; 16(1): 1-6.

\section{GRÁFICO 1 \\ REVASCULARIZZAÇÃO DO MIOCÁRDIO SEM CEC NÚMEROS DE PONTES}

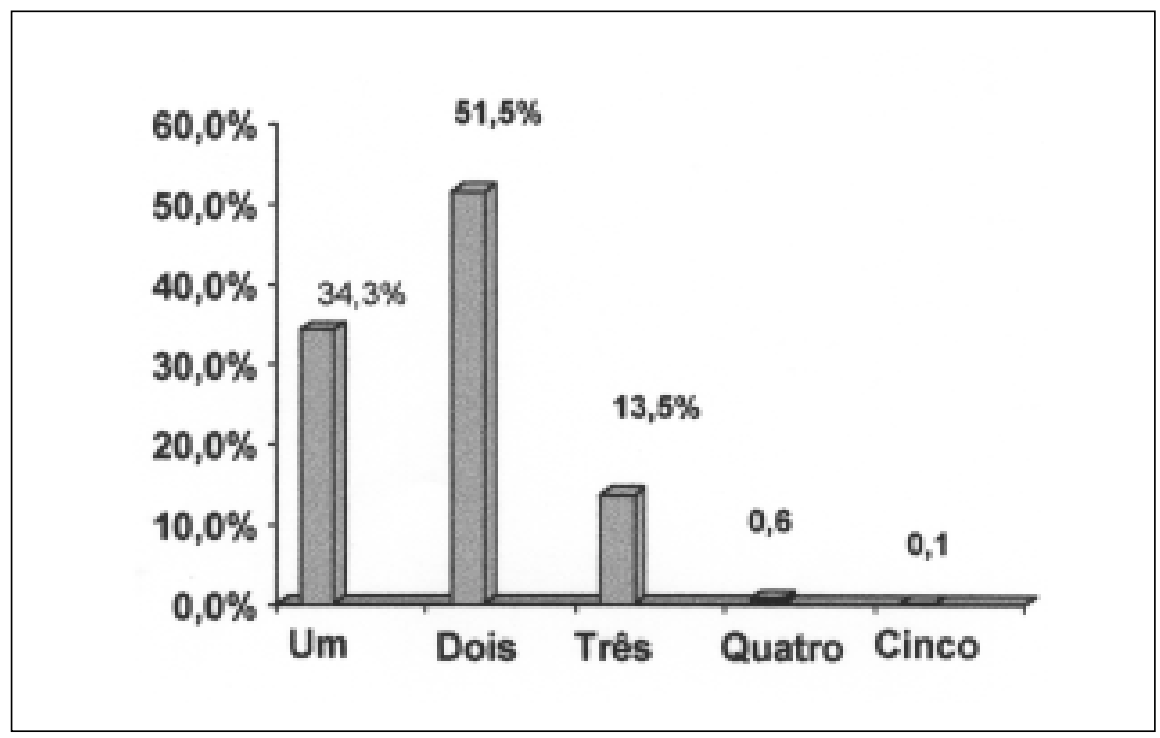

\section{RESULTADOS}

Nesta série de pacientes, a mortalidade glogal, até 30 dias, foi de $1,9 \%$ (48 pacientes), sendo que em sua grande maioria se deram a casos cardíacos, como a síndrome de baixo débito em 1,5 de todos os casos (Tabela 5). Lembramos que 18 pacientes foram para sala de cirurgia em uso de balão intraaórtico e que $20,7 \%$ de todos os casos se encontravam em condições clinicas pré-operatórias de urgência (Tabela 4).

A morbidade apresentada nestes pacientes foi significativamente menor quando comparada ao grupo que faz uso de circulação extracorpórea tanto que a utilização de transfusão sanguínea foi

TABELA 4

REVASCULARIZAÇÃO DO MIOCÁRDIO SEM CEC CONDIÇÕES CLÍNICAS PRÉ-OPERATÓRIAS

Insuficiência Coronária Crônica

Falência de Angioplastias (Aguda ou Crônica)

Reoperações

Angina Instável

Após Trombólise

Infarto em Evolução

18 CASOS COM BIA de $45 \%$ sem CEC em contraste com 90,5\% com CEC. Também contribuiu muito a realização de encisões menores tanto no tórax como nas pernas e abdome (Tabela 2).

\section{TABELA 5}

REVASCULARIZAÇÃO DO MIOCÁRDIO SEM CEC MORTALIDADE HOSPITALAR 48 / $2495(1,9 \%)$

\begin{tabular}{|lc|}
\hline Baixo Débito Cardíaco & 10 \\
\hline Infarto Perioperatório & 7 \\
\hline Morte Súbita & 6 \\
\hline Acidente Vascular Cerebral & 4 \\
\hline Arritmias Primárias & 4 \\
\hline Infecção / Septicemia & 3 \\
\hline Hemorragia & 3 \\
\hline Embolia Pulmonar & 3 \\
\hline Dissecção Aorta & 2 \\
\hline Rotura AAA & 2 \\
\hline Insuficiência Pulmonar & 2 \\
\hline Trombose Mesentérica & 1 \\
\hline Úlcera Gástrica Perfurada & 1 \\
\hline TOTAL & 48 \\
\hline
\end{tabular}


Aguiar L F, Andrade J C S, Branco J N, Palma J H, Teles C A, Gerola L R, Buffolo E - Revascularização do miocárdio sem circulação extracorpórea: resultados da experiência de 18 anos de sua utilização. Rev Bras Cir Cardiovasc 2001; 16(1): 1-6.

Do material avaliado, foi possível averiguar um aumento progressivo da utilização do método que nos últimos 3 anos (36,8\%, 35,1\% e 42\%) em contraste com a aplicabilidade global que foi de $23 \%$ (Tabela 1).

\section{COMENTÁRIOS}

A relização da revascularização direta do miocárdio sem auxílio da circulação extracorpórea foi baixa inicialmente, segundo inumeros autores $(1,2,4)$, porém não teve seguimento pela facilidade da realização das anastomoses com coração parado apesar dos efeitos deletérios da CEC $(3,5,15)$.

A partir de 1985 apresentamos inúmeros pacientes que puderam se beneficiar da técnica $(6,7$, 11), seguida por outros autores que por sua vez aprimoraram os recursos intra-operatórios $(8,9,10)$, fazendo com isso um aumento progressivo nos últimos anos.

Também o uso de estabilizadores, manobras intra-operatórias, pequenas incisões e suas variantes $(9,13,14)$, associados a redução acentuada da morbimortalidade vem incentivando em muito um subgrupo de $42 \%$ dos pacientes submetidos a revascularização do miocárdio nos dias atuais.

RBCCV 44205-523

Aguiar L F, Andrade J C S, Branco J N, Palma J H, Teles C A, Gerola L R, Buffolo E - Myocardial revascularization without extracorporeal circulation: results from 18 years experience. Rev Bras Cir Cardiovasc 2000; 16 (1):1-6.

ABSTRACT: Introduction: Myocardial revascularization without cardiopulmonary bypass is today an increasing alternative of revascularization although the limits of applicability are still to be defined. The authors review a series of cases and discuss its indications based upon their results.

Patients and Methods: There were analyzed 2495 patients who underwent direct myocardial revascularization without extracorporeal circulation in the period from October 1981 to September 1999, from a total of 10656 patients, submitted to coronary bypass surgery during this period $(23.4 \%)$. The age varied from 32 to 90 years (medium $=59$ ) with most males $(67 \%)$. Chronic coronary insufficiency was the most common surgical indication $(70.8 \%)$ and the majority of the patients received 2 grafts (51.5\%).

Results: The global mortality rate (30 days) was $1.9 \%(48 / 2495)$ and only $45 \%$ of these patients needed blood transfusion. The global applicability of this technique was around $23 \%$, however in the last 3 years the applicability was $32.8 \%, 35.1 \%$ and $42.2 \%$.

Conclusions: Myocardial revascularization without extracorporeal circulation is a safe tactical alternative for coronary insufficiency and has increased in the last years. This treatment is indicated in this subgroup of lower mortality and small incidence of postoperative complications. In the next years the use of this technique will increase with the use of stabilizers, special manipulation and functional revascularization.

DESCRIPTORS: Myocardial revascularization, methods. Extracorporeal circulation. Cardiac output, low, sugery. Surgical procedures, minimally invasive. Cardiac surgical procedures, methods.

\section{REFERÊNCIAS BIBLIOGRÁFICAS}

1 Kolessov V I- Mammary artery-coronary artery anastomosis as method of treatment for angina pectoris. $J$ Thorac Cardiovasc Surg 1967; 54: 535-44.

2 Favaloro R G - Saphenous vein autograft replacement of severe segmental coronary artery occlusion: operative technique. Ann Thorac Surg 1968; 5: 334-9.

3 Garrett H E, Dennis E W, DeBakey M E - Aortocoronary bypass with saphenous vein graft: seven-year follow up. JAMA 1973; $223:$ 792-4.
4 Trapp W G \& Bisarya R - Placement of coronary artery bypass graft without pump oxygenator. Ann Thorac Surg 1975; 19: 1-9.

5 Ankeny $\mathrm{J} \mathrm{L} \mathrm{-} \mathrm{To} \mathrm{use} \mathrm{or} \mathrm{not} \mathrm{use} \mathrm{to} \mathrm{the} \mathrm{pump} \mathrm{oxygenator}$ in coronary bypass operations. Ann Thorac Surg 1975; 19: 108-9.

6 Buffolo E, Andrade J C S, Succi J E, Leão L E, Gallucci C - Direct myocardial revascularization without cardiopulmonary bypass. Thorac Cardiovasc. Surg 1985; 33: 26-9. 
Aguiar L F, Andrade J C S, Branco J N, Palma J H, Teles C A, Gerola L R, Buffolo E - Revascularização do miocárdio sem circulação extracorpórea: resultados da experiência de 18 anos de sua utilização. Rev Bras Cir Cardiovasc 2001; 16(1): 1-6.

7 Buffolo E, Andrade J C S, Branco J N R, Aguiar L F, Ribeiro E E, Jatene A D - Myocardial revascularization without extracorporeal circulation: seven-year experience in 593 cases. Eur J Cardiothorac Surg 1990; 4: 504-8.

8 Rivetti L A, Gandra S M A, Pinto e Silva A M R, Campagnucci V P - Revascularização do miocárdio sem circulação extracorpórea com uso de shunt intracardíaco : 12 anos de experiência. Rev Bras Cir Cardiovasc 1997; 12: $226-32$.

9 Jatene F B, Pêgo-Fernandes P M, Assad R S et al. Cirurgia de revascularização do miocárdio minimamente invasiva: resultados com uso de videotoracoscopia e do estabilizador de sutura. Rev Bras Cir Cardiovasc 1997; 12: 233-8.

10 Lobo Fllho J G, Dantas M C B R, Rolim J G V et al. Cirurgia de revascularização completa do miocárdio sem circulação extracorpórea: uma realidade. Rev Bras Cir Cardiovasc 1997; 12: 115-21.

11 Buffolo E, Andrade J C S, Branco J N, Teles C A, Aguiar L F, Gomes W J - Coronary artery bypass grafting

\section{Discussão (Transcriçōes de fita gravada)}

\section{DR. WALDIR JAZBIK}

Rio de Janeiro, $R J$

Quando recebi o convite para comentar este trabalho do grupo do Dr. Enio, na pessoa do Dr. Luciano, achei que eles estavam me convidando para vir elogiar; pois quando tenho dúvida sobre uma palavra, eu vou ao dicionário de língua portuguesa, quando tenho dúvida sobre operação sem circulação extracorpórea, eu consulto a equipe do Dr. Enio.Desta maneira, fica muito difícil eu comentar, mas gostaria de fazer algumas perguntas. Como eu trabalho em hospitais diferentes, um que tem todos os confortos e outro que não. Em um desses hospitais, todos os pacientes são submetidos ao ecocardiograma transesofágico, o que me possibilitou adquirir experiência, de tal forma que, quando eu estou no outro hospital que não tem este exame, posso fazer a mesma observação. E neste particular, tivemos que desenvolver todos os órgãos do sentido, inclusive o olfato. Com isso, nós aprendemos várias coisas, e uma delas é a cor do coração, se ele mudar de cor, não precisa medir fluxo, pode desfazer a anastomose pois ela não está perfeita.Outro aspecto está relacionado à freqüência cardíaca, no começo estávamos muito preocupado com este parâmetro; hoje, os colegas operam com qualquer freqüência, nós também operamos, isto porque não estamos achando solução para diminuí-la. Nossa opinião é without cardiopulmonary bypass Ann Thorac Surg 1996; 61: 63-6.

12 Buffolo E, Andrade J C S, Branco J N R et al. Revascularização do miocárdio sem circulação extracorpórea: analise dos resultados em 15 anos de experiência. Rev Bras Cir Cardiovasc 1996; 11: 227-31.

13 Braile D M, Leal J C F, Soares $M$ J et al. Revascularização do miocárdio com cirurgia minimamente invasiva (MIDCAB): resultados em 46 pacientes. Rev Bras Cir Cardiovasc 1998; 13: 194-7.

14 Jatene F B, Pêgo-Fernandes P M, Hueb A C et al. Revascularização do miocárdio por técnica minimamente invasiva: o que aprendemos após 3 anos com seu emprego. Rev Bras Cir Cardiovasc 1998; 14: 6-13.

15 BRASIL LA - Liberação e efeitos do fator de necrose tumoral alfa (FNT alfa) introduzidos pela circulação extracorpórea [Tese. Mestrado] São Paulo: Escola Paulista de Medicina da Universidade Federal de São Paulo, 1996.

que os anestesistas - eu não deveria falar isto, pois acusar na ausência é covardia, mas talvez uma provocação para que nos próximos congressos eles participem - usam muitas drogas que produzem vasodilatação e os doentes ficam hipovolêmicos; ou eles enchem o doente de água e o preço vem depois no pós-operatório, ou trabalhamos com doente hipovolêmico. Não é só a freqüência cardíaca, mas a maneira com que o coração bate, no paciente hipovolêmico, é insuportável. Às vezes, a freqüência cardíaca não está ruim, mas o batimento cardíaco é desagradável; isto porque os anestesistas não só usam substâncias vasodilatadoras, como também não estão fazendo um pré-anestésico eficiente, o doente entra na sala com freqüência cardíaca alta, com liberação de catecolaminas até na alma, eles começam a intubar e, para compensar está situação, entopem o paciente de drogas e ele fica hipovolêmico. Muitos colegas dizem que operaram o paciente com hipovolemia; na realidade, o doente está com vasoplegia. Neste contexto, gostaria de saber do Dr. Luciano se ele tem esta dificuldade para controlar a freqüência cardíaca, como também a maneira com que o coração bate.

\section{DR. AGUIAR}

Quando soubemos que era o Dr. Jazbik que comentaria nosso trabalho, ficamos muito contentes, pois, com certeza, a opinião dele engran- 
dece muito nosso trabalho. O anestesista é o mesmo em quase todos os lugares, e a brigaa com eles é sempre árdua. Temos um grupo de anestesistas que trabalha conosco há muitos anos e, de certa forma, conseguimos chegar a um meio termo. Dr. Enio sempre conversa com eles e, geralmente, não recebemos o paciente hipovolêmico. Mas nas poucas vezes que isto acontece, o quadro é este que o $\mathrm{Sr}$. falou, os pacientes ficam hipovolêmicos, taquicárdiacos, e isto dificulta muito a realização da ponte. Quando isto acontece, repomos o volume ou criamos algumas manobras para recuperar e, algumas vezes, temos usado Verapamil. Esta é uma droga que temos advogado desde o início de nossa experiência com revascularização sem circulação extracorpórea, e com seu uso conseguimos diminuir o problema. As vezes, só a estabilidade volêmica já é suficiente para que o ritmo se aproxime do normal, que caia a freqüência, para que possamos operar com bastante conforto.

\section{DR. PAULO ROBERTO B. ÉVORA Ribeirão Preto, SP}

Nos nossos pacientes, em geral, que pretendemos operar com ou sem circulação extracorpórea, nós damos $50 \mathrm{mg}$ de Antenolol às 22:00h da noite. Os pacientes chegam com 60, 40 de freqüência, às vezes, se ele está 66 , damos pela manhã junto com o pré-anestésico. Acredito que o betabloqueador, já que não temos Esmolol que permite um controle rígido, é superior ao Verapamil para esta condição de baixar a freqüência.

\section{DR. AGUIAR}

(Encerrando)

Agradeço ao Dr. Évora pelo seu comentário, mas não temos experiência com uso de betabloqieador. Talvez em trabalhos futuros, possamos avaliar este tipo de droga, mas nós atualmente preferimos usar o Verapamil. 